\title{
Biomimetic properties and estimated in vivo distribution of chloroquine and hydroxy-chloroquine enantiomers
}

\author{
Klara L Valko*1 and Tong Zhang ${ }^{2}$ \\ ${ }^{1-}$ Bio-Mimetic Chromatography Ltd, Business \& Technology Centre, Bessemer Drive, Stevenage, Herts SG1 2DX UK \\ ${ }^{2}$ Chiral Technologies Europe, Parc d'Innovation 160, Bd Gonthier d'Andernach CS 8014067404 ILLKIRCH CEDEX \\ France
}

*Corresponding Author: E-mail: klara valko@bio-mimetic-chromatography.com; Tel.: +44-7521-989558;

Received: November 16, 2020; Revised: January 10, 2021; Published online: January 25, 2020

\begin{abstract}
Chloroquine and hydroxy-chloroquine already established as anti-malarial and lupus drugs have recently gained renewed attention in the fight against the Covid-19 pandemic. Bio-mimetic HPLC methods have been used to measure the protein and phospholipid binding of the racemic mixtures of the drugs. The tissue binding and volume of distribution of the enantiomers have been estimated. The enantiomers can be separated using Chiralpak AGP HPLC columns. From the $\alpha$-1-acid-glycoprotein (AGP) binding, the lung tissue binding can be estimated for the enantiomers. The drugs have a large volume of distribution, showed strong and stereoselective glycoprotein binding, medium-strong phospholipid-binding indicating only moderate phospholipidotic potential, hERG inhibition and promiscuous binding. The drug efficiency of the compounds was estimated to be greater than $2 \%$ which indicates a high level of free biophase concentration relative to dose. The biomimetic properties of the compounds support the well-known tolerability of the drugs.
\end{abstract}

C2021 by the authors. This article is an open-access article distributed under the terms and conditions of the Creative Commons Attribution license (http://creativecommons.org/licenses/by/4.0/).

\section{Keywords}

Protein binding; chiral separation; chloroquine and hydroxy-chloroquine; tissue binding; the volume of distribution

\section{Introduction}

Chloroquine (CQ) and hydroxy-chloroquine ( $\mathrm{HCQ}$ ) are well established for the treatment of malaria, rheumatoid arthritis, and lupus. CQ was first synthesised in 1934 by Hans Andersag at Bayer in Elberfeld Germany, by modifying the chemical structure of quinine an anti-malarial natural alkaloid [1]. During the Second World War, when millions of soldiers were taking CQ for malaria prevention, its beneficial effects were discovered in the treatment of rheumatoid arthritis (RA) and systemic lupus erythematosus (SLE). When the resistance of $\mathrm{CQ}$ against malaria plasmids became apparent, the search for analogues resulted in the discovery of HCQ in 1945 [1]. The activity of CQ against viruses was first reported in 1972. by Shimizu et al. [2]. More than ten years ago, CQ was proposed as a broad-spectrum antimicrobial agent against bacteria, fungal and viral infections [3]. In December 2019, an outbreak of pneumonia of unknown aetiology was reported in Wuhan, China $[4,5]$. This disease problem was then declared as a pandemic by 
the World Health Organization (WHO) on the $11^{\text {th }}$ March 2020. The coronavirus was identified as the cause of this respiratory syndrome, and it was named SARS-CoV-2. The disease that it caused was named COVID19. Since then, attention has been given to the use of $C Q$ and $H C Q$ as potential treatments for COVID-19.

The various mechanisms by which $\mathrm{CQ}$ and HCQ may be involved in their antiviral effects have been reviewed recently [6] as a result of increased interest due to the COVID-19 pandemic. Potential mechanisms include prevention of the virus entering the cells, altering virus replication, inhibiting autophagy and modulating the immune response. An interesting antiviral mechanism of CQ and HCQ is that they allow $\mathrm{Zn}$ ions to enter the cell and act as zinc ionophores [7]. Zinc ions exhibit anticancer and antiviral properties by altering the lysosome membrane permeability [8] and the viral RNA-dependent RNA polymerase enzyme and thus inhibit the virus replication process inside the cells.

Figure 1 shows the chemical structures of $\mathrm{CQ}$ and $\mathrm{HCQ}$. HCQ has an additional hydroxyl group that makes the molecule less lipophilic, reducing the severity of side effects and toxicity.

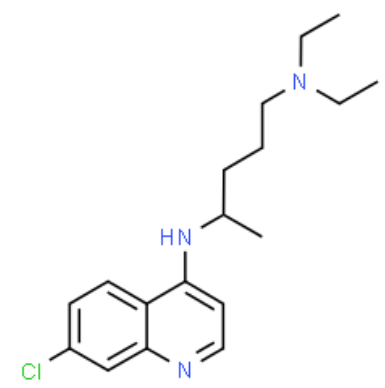

Chloroquine (CQ)

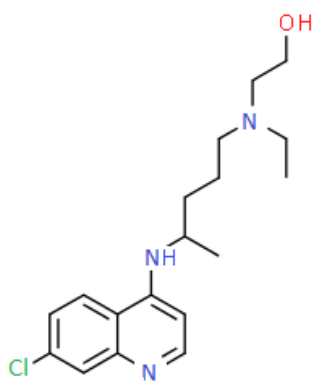

Hydroxy-chloroquine (HCQ)

Figure 1. The chemical structure of chloroquine (CQ) and hydroxy-chloroquine (HCQ)

Both compounds are racemic mixtures, but it has been observed that the enantiomers behave differently in vivo regarding their pharmacokinetics [9] and metabolism [10]. It has been reported that the desethyl $C Q$ excreted by men after treatment with racemic $C Q$ is optically active [11] and that the renal clearance of the racemic $C Q$ drug is a stereoselective process $[9,12,13]$. In vitro efficacy studies did not show any difference between the efficacy of the enantiomers [14], but in vivo (rat) studies have shown a greater efficacy for the (+)-(S)-CQ than the (-)-R-enantiomer $[13,15,16]$. More recent article [17] suggests chiral switches of enantiomers as more potent forms against Covid-19. It may be explained by the stereoselective disposition of $\mathrm{CQ}$ and $\mathrm{HCQ}$ in vivo. Several studies have already been published on the stereoselective blood and plasma protein binding of the two compounds using pure enantiomers [18-20] added to plasma and blood.

The application of chemically bonded chiral stationary phases (Chiralpak HSA and Chiralpak AGP) is a powerful tool for separating racemic mixtures [20]. It was found that the CQ enantiomers differ in their binding to plasma. The (+)-(S)-CQ has a higher binding to human serum albumin (HSA) than the (-)-(R)enantiomer, whereas the latter bound more strongly to $\alpha$-1-acid-glycoprotein (AGP) than the (+)-(S)-CQ. The in vitro human plasma protein binding of $\mathrm{HCQ}$ was also enantioselective: the binding of (+)-(S)-HCQ was almost 2-fold higher than (-)-(R)-HCQ (64\% against $37 \%$ ) [21]. Chromatographic separations of CQ and HCQ and their metabolites have been reported using Chiralpak AGP and Chiralpak AD HPLC columns [18,22], [23]. Other chromatographic methods have been reported for analytical and preparative separations of $C Q$ and HCQ enantiomers [24,25].

Biomimetic HPLC properties can be used to estimate the in vivo distribution (volume of distribution, $V_{\mathrm{d}}$ the unbound volume of distribution, $V_{\text {du }}$ ), drug efficiency and various tissue binding properties of 
compounds [26-28]. The HSA and AGP binding can be measured using Chiralpak HSA and Chiralpak AGP HPLC columns (Chiral Technologies Europe, France). In this way, a different estimation of the in vivo distribution and tissue binding for the different enantiomers can be obtained supposing they provided two requisite peaks from the chromatography, enabling property measurements.

The aim of this study was to measure the biomimetic properties of CQ and HCQ with special emphasis on the different properties of their enantiomers. The other aim was to rationalise the differences in the in vivo potency of the enantiomers that were not detected in vitro. A comparison can then be made of the estimated tissue binding and distribution data with the published in vivo distribution data. The significance of the differences in properties between the $(R)$ and $(S)$ enantiomers can then be evaluated.

\section{Experimental}

Racemic CQ and HCQ of the highest purity were purchased from Merck (Sigma-Aldrich) and were dissolved in DMSO at a $1 \mathrm{mM}$ concentration. The $1 \mathrm{mM}$ solutions were diluted further to $0.1 \mathrm{mM}$ concentration from which $10 \mu \mathrm{L}$ was injected onto the different HPLC columns.

An Agilent 1100 series HPLC system equipped with an autosampler and diode array detector was used for the measurements.

\section{Measurements of lipophilicity at three $\mathrm{pHs}$}

Gemini NX C-18(2) 5 um columns with dimensions of $3 \times 50 \mathrm{~mm}$ (Phenomenex Ltd. Macclesfield, UK) were used for the determination of the Chromatographic Hydrophobicity Index (CHI) and the CHIlogD values [26]. The flow rate was $1.0 \mathrm{~mL} / \mathrm{min}$, with starting mobile phases of $0.01 \mathrm{M}$ formic acid (pH 2.6), $50 \mathrm{mM}$ ammonium acetate adjusted to $\mathrm{pH} 7.4$, and $50 \mathrm{mM}$ ammonium acetate with $\mathrm{pH}$ adjusted to 10.5 to determine the lipophilicity of the compounds at acidic, neutral and alkaline pHs, respectively. An acetonitrile linear gradient was used from 0 to $100 \%$. The acetonitrile concentration reached $100 \%$ in $3.5 \mathrm{~min}$. The $100 \%$ acetonitrile mobile phase was maintained for an additional $1 \mathrm{~min}$ before it was returned to $0 \%$ at $4.7 \mathrm{~min}$. The cycle time of the gradient run was $6 \mathrm{~min}$, with an additional equilibration time of $1 \mathrm{~min}$ before the next injection. The error in the retention time measurements is $\pm 0.005 \mathrm{~min}$ in general after repeated injections.

The set of calibration compounds gave an excellent straight line when their retention time data was plotted against the predefined $\mathrm{CHI}$ values listed in Table 1.

Table 1. The $\mathrm{CHI}$ values of the calibration set of compounds at three $\mathrm{pHs}$ [29]. These values were obtained by fitting the isocratically determined $\mathrm{CHI}$ values and the gradient retention time values. The standard error ranged from 0.1 to $0.8 \mathrm{CHI}$ values. The Chromatographic Hydrophobicity Index (CHI) approximates the acetonitrile concentration when the compound elutes. It can be converted to the octanol/water $\log D$ scale using $\mathrm{CH} I \log \mathrm{D}=0.0525^{*} \mathrm{CHI}-1.467$.

\begin{tabular}{|c|c|c|c|}
\hline Compound & $\mathrm{CHI}$ at $\mathrm{pH} 2.6$ & $\mathrm{CHI}$ at pH 7.4 & $\mathrm{CHI}$ at $\mathrm{pH} 10.5$ \\
\hline Theophylline & 17.9 & 18.4 & 5.0 \\
\hline Phenyl tetrazole & 42.2 & 23.6 & 16.0 \\
\hline Benzimidazole & 6.3 & 34.3 & 30.6 \\
\hline Colchicine & 43.9 & 45.0 & 43.9 \\
\hline Phenyl theophylline & 51.7 & 51.2 & 51.3 \\
\hline Acetophenone & 64.1 & 65.1 & 64.1 \\
\hline Indole & 72.1 & 71.5 & 72.1 \\
\hline Propiophenone & 77.4 & 77.4 & 77.4 \\
\hline Butyrophenone & 87.3 & 87.5 & 87.3 \\
\hline Valerophenone & 96.4 & 96.20 & 96.36 \\
\hline
\end{tabular}


Measurements of phospholipid-binding at pH 7.4 using an Immobilized Artificial Membrane (IAM) column

The phospholipid-binding was measured using an IAM PC.DD2 column with dimensions of $100 \times 4.6 \mathrm{~mm}$ (Regis Technologies Inc., Morton Grove, IL, USA). The gradient retention times of the compounds were measured using a $50 \mathrm{mM}$ ammonium acetate starting mobile phase with the $\mathrm{pH}$ adjusted to 7.4. The mobile phase flow rate was $1.5 \mathrm{~mL} / \mathrm{min}$. The acetonitrile gradient was applied from 0 min to $4.75 \mathrm{~min}$ to reach $90 \%$. The $90 \%$ acetonitrile concentration was maintained for an additional $0.5 \mathrm{~min}$ (to $5.25 \mathrm{~min}$ ), then returned to $0 \%$ by $5.5 \mathrm{~min}$. The cycle time was $6 \mathrm{~min}$ plus an additional $1 \mathrm{~min}$ equilibration time was applied while the injector prepared for the next injection. The gradient retention times were calibrated with the acetophenone homologues for which the $\mathrm{CHI}$ IAM values have been established using isocratic measurements [26]. The Chromatographic Hydrophobicity Index on the IAM column [CHI(IAM)] approximates to the acetonitrile concentration in the mobile phase when the compound elutes. CHI(IAM) values above 45 indicate strong phospholipid binding. Repeating the retention time measurements provided the error only in the third digit of the minute $( \pm 0.005)$.

\section{Measurements of plasma protein binding using Chiralpak HSA and AGP columns}

The protein binding measurements were carried out on Chiralpak HSA and Chiralpak AGP columns with the dimensions of $3 \times 50 \mathrm{~mm}$ with $5 \mathrm{um}$ particle size stationary phase (Chiril Technologies Europe, France). The mobile phase was $50 \mathrm{mM}$ ammonium acetate adjusted to $\mathrm{pH} 7.4$ with a flow rate of $1.2 \mathrm{~mL} / \mathrm{min}$. The standard isopropanol (IPA) gradient up to $35 \%$ was achieved in $3.5 \mathrm{~min}$ and then maintained for $1 \mathrm{~min}$, before returning to $0 \%$ at $4.7 \mathrm{~min}$. The cycle time was $6 \mathrm{~min}$ with an additional $1 \mathrm{~min}$ re-equilibration time. The racemic warfarin showed separation of its enantiomers at retention times of 3.58 and 3.77 mins. The reproducibility of the retention time measurements was within $\pm 0.01 \mathrm{~min}$. The calibration set of compounds and their literature \% binding data that were also converted to log $k$ data are shown in Table 2 . The racemic $C Q$ and $\mathrm{HCQ}$, however, did not show a separation of their enantiomers under such chromatographic conditions and therefore, other gradients and isocratic mobile phases were tested. Figures $2 a$ and Figure $2 b$ show the calibration lines obtained for the HSA and AGP binding, respectively.

\section{The 8 min, "slow gradient" method on the chiral HSA and AGP columns}

To be able to separate the CQ and HCQ enantiomers, the IPA gradient was modified. From 0 to 4 min, the IPA concentration was raised from 0 to $15 \%$, and from 4 to 5 min the $15 \%$ IPA was kept constant before it was reduced to $0 \%$ at $5.2 \mathrm{~min}$. The cycle time was $8 \mathrm{~min}$ plus an additional $1 \mathrm{~min}$ re-equilibration time. The same compounds and data that are listed in Table 2 were used to calibrate the retention times; however, warfarin, diclofenac and isopropanol could not be eluted under this condition.

Table 2. The protein binding data of marketed drug molecules that were used to calibrate the retention times obtained on the chiral protein columns (Chiralpak HSA and Chiralpak AGP). The \% binding data obtained by equilibrium dialysis were converted to $\log k$ data using log $k=\log$ (\%binding/(101-\%binding).

\begin{tabular}{lcccccc}
\hline Compound name & \% HSA & log $\boldsymbol{k}(\mathrm{HSA})$ & $\begin{array}{c}\text { Retention times } \\
\text { HSA }\left(\boldsymbol{t}_{\mathrm{R}}\right)\end{array}$ & \% AGP & log $\boldsymbol{k}(\mathrm{AGP})$ & $\begin{array}{c}\text { Retention times } \\
\text { AGP }\left(\boldsymbol{t}_{\mathrm{R}}\right)\end{array}$ \\
\hline Warfarin & 97.9 & 1.5 & 3.6 & 83.2 & 0.7 & 2.6 \\
Paracetamol & 14.0 & -0.8 & 0.4 & 3.2 & -1.5 & 0.3 \\
Nizatidine & 20.4 & -0.6 & 0.5 & 37.1 & -0.2 & 1.3 \\
Trimethoprim & 37.6 & -0.2 & 1.0 & 46.2 & -0.1 & 1.5 \\
Propranolol & 66.6 & 0.3 & 1.4 & 86 & 0.8 & 2.8 \\
Carbamazepine & 75.0 & 0.5 & 1.6 & 65 & 0.3 & 2.1 \\
Nicardipine & 95.0 & 1.2 & 2.9 & 87 & 0.8 & 2.9 \\
Indomethacin & 99.5 & 1.8 & 4.5 & 56 & 0.1 & 1.8 \\
Diclofenac & 99.8 & 1.9 & 4.3 & 60 & 0.2 & 1.9 \\
\hline
\end{tabular}



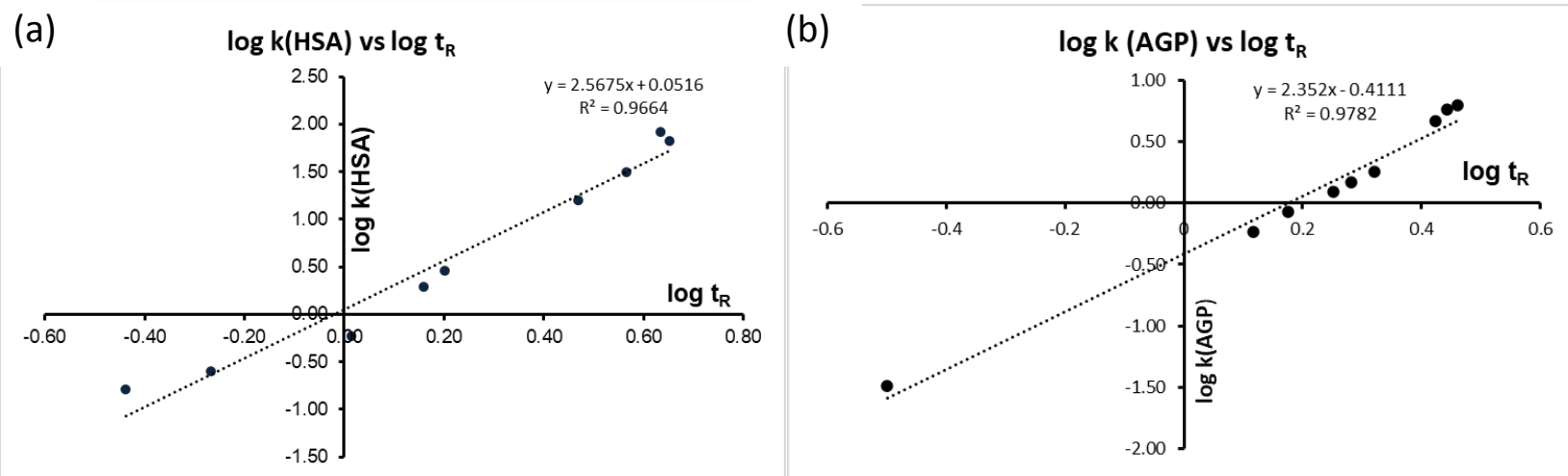

Figure 2. The calibration line obtained on the Chiralpak HSA (a) and Chiralpak AGP (b) columns by plotting the logarithmic value of the obtained retention times and the log $k$ values derived from the literature $\%$ binding data $[30,31]$

\section{Isocratic measurements of CQ and HQ on Chiralpak HSA column}

As the CQ and HCQ enantiomers were not separated using the longer IPA gradient, isocratic analyses were also carried out using a $1.2 \mathrm{~mL} / \mathrm{min}$ flow rate with 5,8 and $10 \%$ IPA, respectively. Using $5 \%$ IPA in the mobile phase, only paracetamol and propranolol eluted from the calibration set of compounds. When $10 \%$ IPA was used warfarin, propranolol and nicardipine were eluted and their respective retention times were used to create a calibration curve for the calculation of the binding of $C Q$ and HCQ from their obtained retention times using the same HPLC conditions.

\section{Measurements of human plasma protein binding by rapid equilibrium dialysis (RED)}

The test compounds were prepared in $100 \%$ pooled human plasma. The measurement was performed using equilibrium dialysis with the two compartments separated by a semi-permeable membrane. The plasma solution was added to one side of the membrane while buffer $(\mathrm{pH} 7.4)$ was added to the other side. The system was allowed to reach equilibrium at $37^{\circ} \mathrm{C}$. Compound concentration on both sides of the membrane was measured by LC-MS/MS, and the fraction of unbound compound calculated. Calibration standards were prepared in plasma and buffer. Test compound incubations were performed in triplicate. A control compound was included in each experiment. The solutions for each batch of compounds were combined into two groups (protein-free and protein-containing). Cassette analysed by LC-MS/MS using two sets of calibration standards for protein-free solutions (7 points) and protein-containing solutions (7 points). The experiments were carried out at Cyprotex Discovery Ltd (UK) and repeated at Sygnature Discovery Ltd (UK) laboratories using their generic LC/MS method. The unbound fraction $\left(f_{\mathrm{u}}\right)$ is calculated from the concentration of the test compound in the protein-containing side $C_{p}$ and the protein-free side $C_{f}$ based on the formula:

$$
f u=1-\left(C_{p}-C_{f}\right) / C_{p} .
$$

\section{Results and Discussion}

The lipophilicity of compounds is an important physicochemical property that may explain the protein binding, the volume of distribution and other distribution properties of drug molecules. It is usually characterised by octanol/water partition $(\log P)$ and distribution $(\log D)$ coefficients. The $\log P$ refers to the neutral form of the molecule, while $\log D$ refers to the compound's distribution between octanol and water at physiological $\mathrm{pH}$ ( $\mathrm{pH}$ 7.4).

The calculated and measured physicochemical properties of $C Q$ and HCQ are listed in Table 3. 
Table 3. Literature and calculated data for $\mathrm{CQ}$ and $\mathrm{HCQ}$

\begin{tabular}{lcc}
\hline Property & CQ & HCQ \\
\hline ACD log $P$ (calculated) & 4.69 & 3.77 \\
Chemaxon log $P$ (calculated) & 3.93 & 2.89 \\
ACD log $D$ (calculated) & 1.74 & 1.96 \\
Chemaxon log D (calculated) & 0.88 & 0.33 \\
Measured log I (ChemSpider [32, 33]) & $2.68-4.63$ & $1.55-3.85$ \\
CHI log D (pH 7.4) & 0.79 & 0.66 \\
CHI log $P$ & 2.68 & 2.13 \\
Chemaxon pKs (calculated) & 10.32 & 9.76 \\
\hline
\end{tabular}

It can be seen in Table 3 that the measured and calculated lipophilicity values vary a lot. In almost all cases, $\mathrm{HCQ}$ is less lipophilic due to the hydrophilic hydroxy group that also affects the basicity of this compound. Comparing the $\log D$ and $\log P$ values, it is apparent that a high percentage of both molecules are

ionised (positively charged) at $\mathrm{pH}$ 7.4. The measured $\mathrm{CHI} \log P$ may well be underestimating the lipophilicity as the $\mathrm{pKa}$ values are around 10 . Therefore, the mobile phase applied pH 10.5 may not suppress the ionisation of both molecules completely, and the actual lipophilicity of the neutral form of these molecules can be much greater.

Table 4 shows the measured phospholipid and protein binding data of the two compounds obtained using the standard 6 min protocol [26].

It can be seen from Table 4 that $\mathrm{HCQ}$ has marginally weaker binding to phospholipids and plasma proteins. The enantiomers could not be separated on the chiral HSA and AGP stationary phases using the standard high-throughput protocol for biomimetic property measurements. The phospholipid-binding is in a medium-strong range. It has been reported that compounds that have $\mathrm{CHI}$ IAM values greater than 50 have the potential for promiscuous binding and causing phospholipidosis [34]. The hERG inhibition, which is an indicator for causing arrhythmia and heart condition, is also associated with lipophilic and positively charged compounds [35]. Positively charged compounds usually have stronger binding to IAM than isolipophilic compounds without the presence of positive charge [36]. However, the CHI IAM values of CQ and $\mathrm{HCQ}$ are below 50. The measured protein binding was greater than has been published [19], ranging from 46 to $74 \%$ in patients with rheumatoid arthritis. Strong AGP binding was also observed, being approximately the same as the albumin binding.

Table 4. The measured phospholipid binding (CHI IAM), protein binding and estimated total plasma protein binding (PPB) based on the \% HSA and \% AGP binding and the lipophilicity of the compounds. The error of the values depends on the model error and the error obtained from fitting the calibration lines. The CHI IAM values are reproducible with $\pm 3 \mathrm{CHI}$ unit, the protein binding \% error is larger at the middle range ( $\pm 10 \%$ ) and much smaller above $90 \%$ binding $( \pm 0.1 \%)$, the estimation of the total plasma protein binding includes the model error of \pm 0.36 in log $k$ PPB values.

\begin{tabular}{lcc}
\hline Property & CQ & HCQ \\
\hline CHI (IAM) & 47 & 45.4 \\
$\log k$ (IAM) & 2.6 & 2.5 \\
\% HSA bound & 87.6 & 84.9 \\
$\log k$ HSA & 0.8 & 0.7 \\
\% AGP bound & 88.8 & 89.0 \\
log $k$ AGP & 0.9 & 0.9 \\
Estimated \% plasma protein binding (PPB) & 94.8 & 93.4 \\
log $k$ PPB & 1.2 & 1.1 \\
\hline
\end{tabular}

However, the AGP concentration in the plasma ranges from 1 to $10 \%$ while the albumin concentration in the plasma is around $60 \%$, being the most abundant protein. It is also well established that AGP prefers to bind to positively charged compounds since it has negatively charged sialic acids at its binding site [37]. The lysosomes inside the cell also contain glycoproteins that are negatively charged and attract positively 
charged compounds. The strong AGP binding of CQ and HCQ may explain the large variability of the plasma protein binding data as the AGP concentration depends on the disease state and it can increase significantly in inflammation, cancer and with age [38].

Table 5 shows the equations of the models that were used to make estimations of the in vivo properties of the drugs from the measured biomimetic HPLC data. Note, that the $\log k$ HSA and $\log k$ IAM data had to be transformed to log $K$ HSA and log $K$ IAM data as the measured log $k$ data for acetophenone homologues up to octanophenone did not show linear correlation with their octanol water partition coefficients $(\log P)$ [39]. For the mechanistic model of the volume of distribution, we converted both the measured albumin and phospholipid binding data so that they showed linear correlation with the linear free-energy related $\log P$ values. Thus, the regression coefficients in linear regression analysis are meaningful and reflect to the physiological proportion of the albumin and phospholipids in the body. The estimated distribution and tissue binding data for $\mathrm{CQ}$ and $\mathrm{HCQ}$ were evaluated using the standard 6-min HPLC based measurements of biomimetic properties (CHIlog $D$ at three $\mathrm{pHs}, \log k \mathrm{HSA}, \log k$ AGP and $\log k$ IAM). They are based on the calibrated retention times obtained on C-18, IAM, Chiralpak HSA and Chiralpak AGP columns. The total plasma protein binding, lung tissue binding, drug efficiency, brain tissue binding and brain to blood ratio, cell partition coefficient and lung tissue binding have been calculated using the equations listed in Table 5 and are shown in Table 6.

Table 5. The published and validated model equations that are used together with the measured properties to estimate the in vivo distribution behaviour of drugs. (abbreviations: IAM = Immobilized Artificial Membrane; HSA = Human Serum Albumin; $V_{d}=$ volume of distribution; $V_{\mathrm{du}}=$ Unbound volume of distribution, $D E_{\max }=$ maximum drug efficiency; $\mathrm{BTB}=$ brain tissue binding; $\mathrm{PPB}=$ plasma protein binding, $f_{\mathrm{u}}$ $=$ unbound fraction; $K_{\mathrm{bb}}=$ brain to blood partition coefficient; $K_{\text {pcell }}=$ cell partition coefficient; $\mathrm{LTB}=$ lung tissue binding)

\begin{tabular}{|c|c|}
\hline $\log K$ IAM [43] & $=0.29 * \mathrm{e}(0.026 \mathrm{CHI}(\mathrm{IAM})+0.42)+0.7$ \\
\hline $\log k$ IAM [43] & $=0.046 * \mathrm{CHI}(\mathrm{IAM})+0.42$ \\
\hline $\begin{array}{l}\text { Phospholipidotic potential (CAD- } \\
\text { likeness) }\end{array}$ & $=\mathrm{CHI}(\mathrm{IAM})+(\mathrm{CHI}$ at $\mathrm{pH} 10.5-\mathrm{CHI}$ at $\mathrm{pH} 7.4)$ \\
\hline $\log K \mathrm{HSA}[43]$ & $=\operatorname{elog} k(\mathrm{HSA})$ \\
\hline $\log k \mathrm{HSA}[30]$ & $=\log (\% H S A b o u n d /(101-\% H S A$ bound $))$ \\
\hline Estimated $\log V_{d}[43,44]$ & $=0.44 * \log K$ IAM $-0.22 * \log K \mathrm{HSA}-0.62$ \\
\hline Estimated $\log V_{\mathrm{du}}[27]$ & $=0.23 * \log K \mathrm{HSA}+0.43 * \log K \mathrm{IAM}-0.72$ \\
\hline$D E_{\max }[45]$ & $=100 / V_{\mathrm{du}}$ \\
\hline $\log k$ BTB [31] & $=1.29 * \log k \mid A M+1.03 * \log k \mathrm{HSA}-2.37$ \\
\hline $\log k(\mathrm{PPB})[31]$ & $=0.98 * \log k H S A+0.19 * \log k A G P+0.031 * \mathrm{CHI} \log D 7.4-0.20$ \\
\hline$\%$ BTB [31] & $=100 * 10 \log k \mathrm{BTB} /(1+10 \log k \mathrm{BTB})$ \\
\hline \%РPB [31] & $=100 * 10 \log k \mathrm{PPB} /(1+10 \log k \mathrm{PPB})$ \\
\hline$f_{\mathrm{u}} \mathrm{BTB}$ and PPB [31] & $=(100-\% \mathrm{BTB}) / 100$ and $(100 \%-\%$ PPB $) / 100$ \\
\hline$K_{\mathrm{bb}}[31]$ & $=f_{\mathrm{u}} \mathrm{PPB} / f_{\mathrm{u}} \mathrm{BTB}$ \\
\hline $\log K_{\text {pcell }}[46]$ & $=1.1 \log k \mathrm{IAM}-1.9$ \\
\hline $\log k$ LTB [31] & $=0.49 * \log k \mathrm{PPB}+0.34 \mathrm{CH} \log D-0.069$ \\
\hline$\%$ LTB [31] & $=100 * 10^{\log k L T B} /\left(1+10^{\log k L T B}\right)$ \\
\hline
\end{tabular}

It can be seen from Table 6 that both compounds have CAD-likeness values greater than 50, which indicates that both have phospholipidotic potential. It is known that chloroquine causes phospholipidosis both in animals and in human [40-42]. However, the phospholipidotic potential of HCQ is much less than $\mathrm{CQ}$. Although the phospholipid-bindings (CHI IAM) were not very different, the greater difference in CADlikeness values is due to the larger percentage of the positively charged form of CQ than in HCQ. It is obvious from the lower acid dissociation constant of $\mathrm{HCQ}$ and the smaller difference between the CHIlogP 
and $\mathrm{CH}$ llogD values (see Table 3). It also implies that HCQ is less likely to cause hERG inhibition and promiscuous binding and in general, fewer side effects. Based on the measured data, the expected brain tissue binding did not show a significant difference between $\mathrm{CQ}$ and $\mathrm{HCQ}$. On the contrary, the brain to blood ratio is smaller in HCQ than CQ due to the slightly stronger AGP binding of HCQ that keeps the compound in the plasma compartment.

Table 6. The estimated biomimetic properties of $\mathrm{CQ}$ and $\mathrm{HCQ}$

\begin{tabular}{lcc}
\hline Property & CQ & HCQ \\
\hline Phospholipidotic potential & 82.8 & 73.6 \\
(CAD-likeness) & 98.4 & 97.6 \\
\%BTB & 0.9 & 0.8 \\
est log k BB & 3.4 & 2.8 \\
Brain to plasma & 6.9 & 5.1 \\
$V_{\mathrm{d}}(\mathrm{L} / \mathrm{kg})$ & 56.1 & 38.8 \\
$V_{\mathrm{du}}(\mathrm{L} / \mathrm{kg})$ & 1.8 & 2.6 \\
$D E_{\mathrm{max}} \%$ & 96.3 & 93.9 \\
$\% \mathrm{LTB}$ & 8.7 & 7.3 \\
$\mathrm{~K}_{\text {pcell }}$ & & \\
\hline
\end{tabular}

Interestingly the expected volume of distribution based on the measured biomimetic properties is 6.9 and $5.8 \mathrm{~L} / \mathrm{kg}$ for $\mathrm{CQ}$ and $\mathrm{HCQ}$, respectively, which is not as high as over $100 \mathrm{~L} / \mathrm{kg}$ as has been described in the literature $[16,47-$ 49]. The $V_{d}$ of CQ was 116 to $285 \mathrm{~L} / \mathrm{kg}$ in healthy volunteers while $\mathrm{HCQ}$ was reported to have a much higher volume of distribution at above $4000 \mathrm{~L} / \mathrm{kg}$ [48]. Tett et al. [50], reported a range of steady-state $V_{\mathrm{d}}$ values for HCQ ranging from

2402 to $8346 \mathrm{~L}$ that are equivalent to 34.3 to $119 \mathrm{~L} / \mathrm{kg}$. The volume of distribution was 5 to 7 times greater when the plasma concentration was considered instead of the blood concentration as the blood/plasma ratio was reported to be 5 to 7 . The reported plasma protein binding value that was determined in 1986 was $66.6 \%$ [20]; enantioselective plasma protein binding, albumin and AGP binding were also reported. The human plasma protein binding measurements were repeated using the rapid equilibrium dialysis method and the obtained average values were $52.6 \%$ ( $\pm 6.2 \%)$ and $30.7 \%$ ( $\pm 11.3 \%)$ for CQ and HCQ, respectively. The estimated plasma protein binding based on the biomimetic HPLC measurements was much stronger ( $95 \%$ and $93 \%$ for $\mathrm{CQ}$ and $\mathrm{HCQ}$, respectively) than that published using in vivo data. At this stage, there is no explanation for the difference. This is the cause of the much lower estimated volume of distribution.

The AGP binding using equilibrium dialysis method was reported to be $48.4 \%$ (59.9 and $34.9 \%$ of the R and S enantiomers, respectively) for CQ and $34 \%$ ( 29 and $41 \%$ for the R and S enantiomer, respectively) for HCQ [51]. The albumin binding was only 38.6 and $40 \%$ for $C Q$ and $H C Q$, respectively, by the same author [51]. These values were quoted from earlier publications where the actual measurements were originally reported [52-54]. It was observed that under the standard IPA gradient conditions, both CQ and HCQ eluted between Carbamazepine and Nicardipine, being closer to Nicardipine, which binds to plasma proteins at 75 and $95 \%$, respectively. This explains why the HSA binding was calculated as being 87 and $83 \%$ (see Table 3). The $D E_{\max }$ values reveal the free plasma concentration relative to the dose. Most marketed drug molecules have $D E_{\max }$ values of around $1 \%$. It can be seen that both CQ and HCQ have greater than $1 \%$ drug efficiency, which indicates that a high free concentration can be achieved with a relatively low dose. The estimated lung tissue binding for both $C Q$ and $\mathrm{HCQ}$ was weaker than their brain tissue binding. As mucus binding correlates very well with the AGP binding, strong mucus binding can be expected for both compounds. The cellular partition coefficients of both compounds are high ( $K_{\text {pcell }}$ above 7$)$, which means a 7 to 8 times greater concentration than the intracellular concentration can be expected for both compounds.

Unfortunately, our standard fast gradient protocols used for the HSA and AGP binding measurements did not allow the separation of the enantiomers of $\mathrm{CQ}$ or $\mathrm{HCQ}$. This is also an indication that there are no significant differences in the biomimetic properties of each respective enantiomer. Therefore, new HPLC 
conditions were evaluated on the Chiralpak HSA and Chiralpak AGP column by increasing the gradient analysis time and reducing the maximum IPA concentration in the mobile phase. Slowing down the gradient to reach 10 and $20 \%$ IPA did not result in the separation of the enantiomers on the HSA column, and both $\mathrm{CQ}$ and HCQ eluted after Carbamazepine (75\% plasma protein binding). The calculated HSA binding was 82.2 and $81.9 \%$ for $\mathrm{CQ}$ and $\mathrm{HCQ}$, respectively. Isocratic measurements using 5, 8 and $15 \%$ IPA in the mobile phase resulted in $80 \%$ binding when the relative retention times obtained using a partial set of calibration compounds were taken into consideration. No separation of the enantiomers was observed. Figure 3 shows the chromatograms of $C Q$ and HCQ obtained on a ChiralPak HSA column using \%IPA in isocratic mode. However, no separation of enantiomers was observed.

(a)

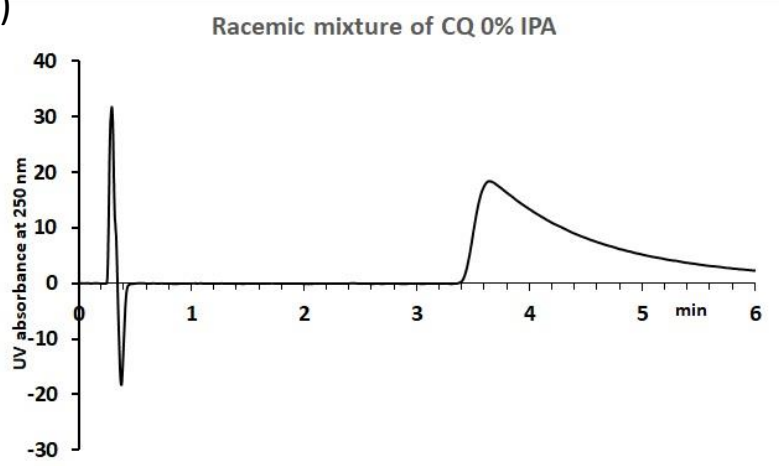

(b)

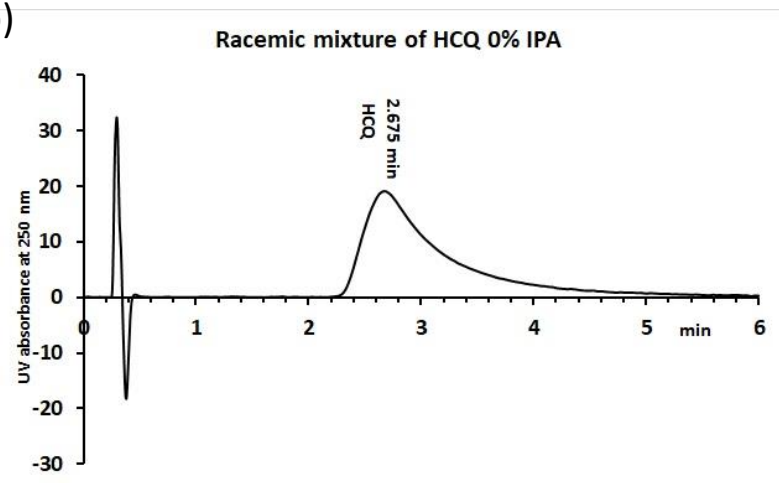

Figure 3. The chromatograms of CQ (a) and HCQ (b) on ChiralPak HSA column using $0 \%$ IPA in the mobile phase in isocratic mode.

When similar changes in the mobile phases were applied using the Chiralpak AGP column, excellent separations of both the $C Q$ and $H C Q$ enantiomers were observed albeit with a very short column size ( $L=50$ $\mathrm{mm}$ ) and fast flow rate $(1.2 \mathrm{~mL} / \mathrm{min})$. It can be seen in Figure 4 that the separation of enantiomers occurred under the shallower gradient conditions. Still, no significant differences between the retention times were observed for CQ and HCQ confirming that the AGP binding of the two molecules is quite similar. The chiral separations were also obtained in the isocratic mode. However, the difference in binding between the enantiomers was only 3 to $4 \%$. Based on these measurements, the AGP binding is much stronger for both $\mathrm{CQ}$ and $\mathrm{HCQ}$ than previously reported. When the AGP binding is above $80 \%$, the compounds are expected to have variable efficacy in the clinic. It is because the AGP concentration changes in various disease states and it can alter the total plasma protein binding significantly, and that may cause a reduction of the free therapeutic concentration of the drugs.

(a)

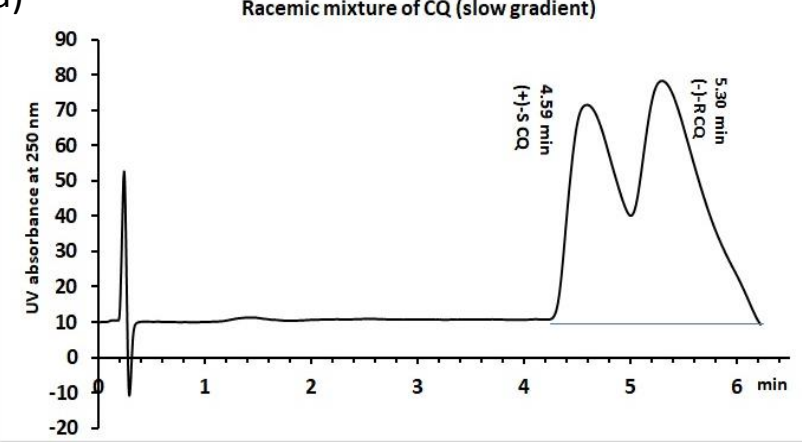

(b)

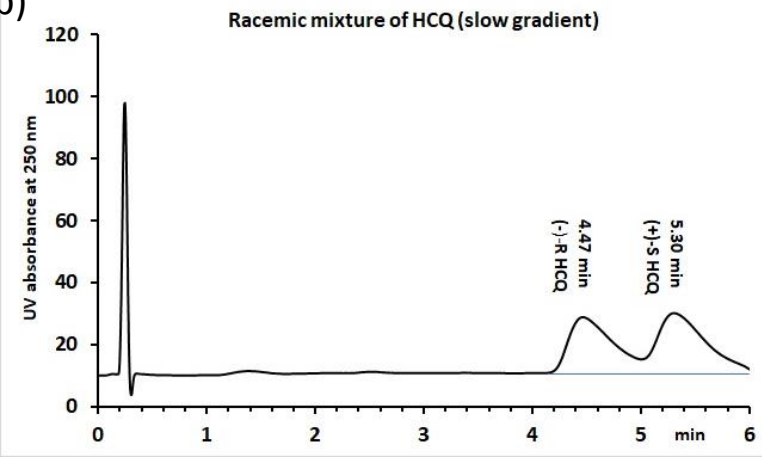

Figure 4. The chromatogram of racemic Chloroquine (CQ) (a) and hydroxychloroquine (HCQ) (b) obtained using the shallower gradient method ("slow gradient") with 0 to $15 \%$ IPA over 4.0 min and then maintained at $15 \%$ for $1 \mathrm{~min}$ before returning to $0 \%$ IPA on a Chiralpak AGP column $(50 \times 3 \mathrm{~mm})$ with a $1.2 \mathrm{ml} / \mathrm{min}$ flow rate. The enantiomers are identified based on ref [51] that reported $C Q(R)$ and $H C Q(S)$ as the stronger binders to AGP. 
Table 7 shows the \% binding data obtained using a slow gradient (up to $15 \%$ IPA). The protein binding measurements were repeated using isocratic conditions with 5, 8 and $15 \%$ IPA in the mobile phase. Under such conditions, stronger binding compounds from the calibration mixture (warfarin, indomethacin and diclofenac) could not be eluted. The retention times of CQ and HCQ obtained when using 5,8 and $15 \%$ IPA on Chiralpak HSA and Chiralpak AGP columns are listed in Table 8.

Table 7. The measured and estimated biomimetic properties of $C Q$ and HCQ based on the data obtained using the slower and shallower gradient (up to $15 \%$ IPA).

\begin{tabular}{lcccc}
\hline Property & (+)-S CQ & (-)-R CQ & (+)-S HCQ & (-)-R HCQ \\
\hline \% bound HSA & 82.2 & 82.2 & 81.9 & 81.9 \\
\% bound AGP & 85.0 & 88.4 & 88.5 & 84.3 \\
calc\%PPB & 90.6 & 91.1 & 91.2 & 91.7 \\
CHI log $D_{2}$ & -1.2 & -1.2 & -1.3 & -1.3 \\
CHI log $D_{7.4}$ & 0.8 & 0.8 & 0.7 & 0.7 \\
CHI log $D_{10.5}$ & 2.7 & 2.7 & 2.1 & 2.1 \\
CHIlog $P$ & 2.7 & 2.7 & 2.1 & 2.1 \\
CHI IAM & 46.9 & 46.9 & 45.4 & 45.4 \\
CAD-likeness & 82.9 & 82.9 & 73.6 & 7367 \\
\%BTB & 97.6 & 97.6 & 97.1 & 97.0 \\
est log $k$ BB & 0.9 & 0.9 & 0.9 & 0.8 \\
Brain to plasma & 4.0 & 3.8 & 3.0 & 2.8 \\
$V_{\mathrm{d}}$ L/kg & 8.2 & 8.2 & 6.4 & 6.3 \\
$V_{\text {du }}$ & 45.7 & 45.8 & 35.3 & 35.1 \\
$D E_{\text {max }} \%$ & 2.2 & 2.2 & 2.8 & 2.9 \\
$\%$ lung tissue binding & 95.5 & 95.6 & 93.1 & 93.3 \\
\hline
\end{tabular}

Table 8. The retention times obtained for CQ and HCQ (enantiomers) using a $1.2 \mathrm{~mL} / \mathrm{min}$ flow rate and 5, 8 and 15 $\%$ IPA on Chiralpak HSA and Chiralpak AGP columns. The calculated \% binding data are also given in brackets. The retention times of Propranolol and Nicardipine, the two basic compounds from the calibration set, are also shown. The last two rows in the table are the data from reference [51] where the values were obtained using an equilibrium dialysis method ( $\mathrm{n} / \mathrm{a}$ means not applicable)

\begin{tabular}{lcccccc}
\hline Compound/Cond. & CQ (R) & CQ (S) & HCQ (R) & HCQ (S) & $\begin{array}{c}\text { propranolol } \\
\text { (66 \% PPB) }\end{array}$ & $\begin{array}{c}\text { nicardipine } \\
\text { (96 \% PPB) }\end{array}$ \\
\hline HSA 5 \% IPA (min) & $0.89(60 \%)$ & $1.15(68 \%)$ & $0.94(61 \%)$ & $1.4(73 \%)$ & $0.76(54 \%)$ & $6(96 \%)$ \\
HSA 8 \% IPA (min) & $0.87(64 \%)$ & $0.87(64 \%)$ & $0.91(66 \%)$ & $0.91(66 \%)$ & $0.73(57.6 \%)$ & $3.39(95 \%)$ \\
HSA 15 \% IPA (min) & $0.68(80 \%)$ & $0.68(80 \%)$ & $0.69(80 \%)$ & $0.69(80 \%)$ & $0.58(66 \%)$ & $1.05(95 \%)$ \\
AGP 5 \% IPA (min) & $3.84(86 \%)$ & $5.24(90 \%)$ & $3.84(86 \%)$ & $5.4(90 \%)$ & $2.78(85 \%)$ & $>8$ min (>98\%) \\
AGP 8 \% IPA (min) & $2.53(84 \%)$ & $2.55(84 \%)$ & $2.95(85 \%)$ & $3.85(87 \%)$ & $1.73(68 \%)$ & $5.63(98 \%)$ \\
AGP 15 \% IPA (min) & $2.20(82 \%)$ & $2.00(81 \%)$ & $1.98(81 \%)$ & $2.23(83 \%)$ & $1.58(67 \%)$ & $5.0(96 \%)$ \\
\% HSA bound [51] & $30 \%$ & $54 \%$ & $50 \%$ & $29 \%$ & $n / a$ & $n / / a$ \\
\hline
\end{tabular}

It can be seen in Table 8 that the estimated \% binding data using the retention time measured for the partial calibration set of compounds changes depending on the experimental conditions. The HSA binding ranged from 60 to $80 \%$ but generally was much greater than the published albumin (HSA) and alpha-1-acid glycoprotein (AGP) binding data. This is because the retention times dependency on the IPA concentration was greater for the calibration set of compounds than for the CQ and HCQ. Table 9 shows the measured 
and estimated biomimetic properties of $\mathrm{CQ}$ and $\mathrm{HCQ}$ based on the data obtained when using the isocratic method on the Chiralpak HSA and Chiralpak AGP column (IPA concentration $15 \%$ ). Probably, CQ and HCQ do not bind to the hydrophobic site on the albumin but could bind to a specific area that is not affected strongly by the IPA concentration. The AGP binding was also much stronger using the biomimetic methodology that converts the $\%$ binding to the total plasma protein concentration where the AGP concentration is only 1 to $3 \%$. The difference can be explained by the different scaling used when expressing the \% of the compound bound in plasma or to a single protein. However, when using the lowest IPA concentration in the mobile phase (5\%), the data obtained was between 77 and $87 \%$ for the enantiomers of $\mathrm{CQ}$ and $\mathrm{HCQ}$. The in vivo PK data also showed large individual variations in humans which suggests that these compounds may bind to a particular type of negatively charged proteins in plasma and in tissues that may have large concentration variations.

Table 9. The estimated in vivo properties of $C Q$ and HCQ enantiomers obtained from the data when the protein binding was obtained with the $15 \%$ IPA isocratic conditions.

\begin{tabular}{|c|c|c|c|c|}
\hline Property & $(+)-S C Q$ & $(-)-R C Q$ & (+)- S HCQ & (-)-R HCQ \\
\hline$\%$ bound HSA & 79.9 & 79.9 & 79.9 & 79.9 \\
\hline$\%$ bound AGP & 82.0 & 81.0 & 85.1 & 87.3 \\
\hline calc\% PPB & 89.9 & 89.7 & 89.0 & 88.9 \\
\hline $\mathrm{CHI} \log \mathrm{D}_{2}$ & -1.2 & -1.2 & -1.3 & -1.3 \\
\hline $\mathrm{CHI} \log \mathrm{D}_{7.4}$ & 0.8 & 0.8 & 0.7 & 0.7 \\
\hline $\mathrm{CHI} \log \mathrm{D}_{10.5}$ & 2.7 & 2.7 & 2.1 & 2.1 \\
\hline CHIlogP & 2.68 & 2.7 & 2.1 & 2.1 \\
\hline $\mathrm{CHI}$ IAM & 46.92 & 46.9 & 45.4 & 45.4 \\
\hline CAD-likeness & 82.89 & 82.9 & 73.6 & 73.6 \\
\hline \%ВТВ & 97.26 & 97.3 & 96.7 & 96.7 \\
\hline est $\log k$ BB & 0.96 & 1.0 & 0.9 & 0.9 \\
\hline Brain to plasma & 4.06 & 4.0 & 3.1 & 3.0 \\
\hline $\log V_{d}$ & 0.94 & 0.9 & 0.8 & 0.8 \\
\hline$V_{\mathrm{d}} \mathrm{L} / \mathrm{kg}$ & 8.65 & 8.7 & 6.7 & 6.6 \\
\hline $\log V_{\mathrm{du}}$ & 1.63 & 1.6 & 1.5 & 1.5 \\
\hline$V_{\mathrm{du}}$ & 43.01 & 43.0 & 33.5 & 33.3 \\
\hline$D E_{\max } \%$ & 2.33 & 2.3 & 23.0 & 3.0 \\
\hline$\%$ lung tissue binding & 96.05 & 95.1 & 92.6 & 92.7 \\
\hline
\end{tabular}

It can be seen that the estimated distribution data shown in Table 7 and Table 9 are very similar, and do not differ significantly despite the large differences in the \% protein binding data. It is important to note that the \% binding data are not linearly related to the protein binding partition coefficients. Still, they have a sigmoidal relationship with the log $k$ and logarithmic retention time data. This means that small variations in the log $k$ HSA and log $k$ AGP values result in a large variation when expressed as percentage binding in the range of 30 to $80 \%$. In the stronger binding range, large variations in retention times will result in only a small per cent of change. The biomimetic protein binding data have been validated for differentiating between strong binders and are more accurate in that region [30].

Conflict of interest: Klara Valko is the founder of BioMimetic chromatography LTD and Tong Chang is a scientist at Chiral Technologies Europe.

\section{Conclusions}

CQ and HCQ have gained renewed interest as potential cures for COVID-19 when used in combination with antibiotics and zinc. Biomimetic properties derived from biomimetic HPLC stationary phases have 
never been measured for these compounds. $C Q$ and HCQ did not show extreme phospholipid-binding that would give the compounds a negative toxicity profile. CQ and HCQ bind to AGP very strongly and stereoselectively, though there is no significant difference between $C Q$ and HCQ in this regard. The stereoselective binding to AGP did not result in significant differences in the estimated total plasma protein binding, lung tissue binding and volume of distribution. The retention time measurements are very reproducible on the biomimetic columns, but the absolute values of the calculated binding data originate from the error of the calibration line fit. However, the order of the retention data is very reproducible. The in vivo distribution models include 0.5 to 1 log unit errors but again the rank order of the compounds is reproducible. The comparison between the estimated distribution properties and the properties published in the literature show great variations, however the biomimetic protein binding data were greater than the measured plasma protein binding data obtained by equilibrium dialysis. The aim of the study was to differentiate the binding of the various enantiomers and estimate the in vivo distribution of each enantiomer. It was found that the small differences in the protein binding data did not manifest itself in significant differences in the predicted in vivo distribution data, so this cannot explain the observed differences in the in vivo activity of the enantiomers. It was found that the drugs bind to glycoproteins that are present in humans in variable amounts from various disease states, which may explain the big variations observed in the in vivo distribution and protein binding data.

\section{References}

[1] K. Krafts, E. Hempelmann, A. Skórska-Stania. From methylene blue to chloroquine: A brief review of the development of an antimalarial therapy. Parasitol. Res. 111 (2012) 1-6. doi: https://doi.org/10.1007/s00436-012-2886-x.

[2] Y. Shimizu, S. Yamamoto, M. Homma, N. Ishida. Effect of chloroquine on the growth of animal viruses. Arch. Gesamte Virusforsch. 36 (1972) 93-104. doi: https://doi.org/10.1007/BF01250299.

[3] J.M. Rolain, P. Colson, D. Raoult. Recycling of chloroquine and its hydroxyl analogue to face bacterial, fungal and viral infections in the 21st century. Int. J. Antimicrob. Agents 30 (2007) 297-308. doi: https://doi.org/10.1016/i.ijantimicag.2007.05.015.

[4] H. Lu, C.W. Stratton, Y.W. Tang. Outbreak of pneumonia of unknown etiology in Wuhan, China: The mystery and the miracle. J. Med. Virol. 92 (2020) 401-402. doi: https://doi.org/10.1002/jmv.25678.

[5] R. Lu, X. Zhao, J. Li, P. Niu, B. Yang, H. Wu, W. Wang, H. Song, B. Huang, N. Zhu, Y. Bi, X. Ma, F. Zhan, L. Wang, T. Hu, H. Zhou, Z. Hu, W. Zhou, L. Zhao, J. Chen, Y. Meng, J. Wang, Y. Lin, J. Yuan, Z. Xie, J. Ma, W.J. Liu, D. Wang, W. Xu, E.C. Holmes, G.F. Gao, G. Wu, W. Chen, W. Shi, W. Tan. Genomic characterisation and epidemiology of 2019 novel coronavirus: implications for virus origins and receptor binding. Lancet 395 (2020) 565-574. doi: https://doi.org/10.1016/S0140-6736(20)30251-8.

[6] T.J. Oscanoa, R. Romero-Ortuno, A. Carvajal, A. Savarino. A pharmacological perspective of Chloroquine in SARS-CoV-2 infection. Int. J. Antimicrob. Agents 56 (2020) 106078. doi: https://doi.org/10.1016/J.IJANTIMICAG.2020.106078.

[7] J. Xue, A. Moyer, B. Peng, J. Wu, B.N. Hannafon, W.Q. Ding. Chloroquine is a zinc ionophore. PLoS One 9 (2014) 1-6. doi: https://doi.org/10.1371/journal.pone.0109180.

[8] J. Zheng, X.X. Zhang, H. Yu, J.E. Taggart, W.Q. Ding. Zinc at cytotoxic concentrations affects posttranscriptional events of gene expression in cancer cells. Cell. Physiol. Biochem. 29 (2012) 181188. doi: https://doi.org/10.1159/000337599.

[9] H. Fieger, J. Iredale, I.W. Wainer. Enantioselective determination of hydroxychloroquine and its major metabolites in urine and the observation of a reversal in the $(+) /(-)$-hydroxychloroquine ratio. Chirality 5 (1993) 65-70. doi: https://doi.org/10.1002/chir.530050205.

[10] Y. Wei, G.A. Nygard, S.L. Ellertson, S.K.W. Khalil. Stereoselective disposition of hydroxychloroquine and its metabolites in rats. Chirality 7 (1995) 598-604. doi: https://doi.org/10.1002/chir.530070807. 
[11] C.M. Eldefield E.O. Titus, L.C. Craig, C. Golumbic, H. R. Highton. Identification by distribution studies; application to metabolic studies of 4-aminoquinoline antimalarial. J. Org. Chem. 13 (1948) 39-62.

[12] H.S.D. Ofori-Adjei, O. Ericson, B. Lindström, J. Hermansson, K. Adjepon-Yamoah. Enantioslective analysis of chloroquine and desethylchloroquine after oral administration of racemic chloroquine. Ther. Drug Monit. 8 (1986) 457-461. https://doi.org/10.1097/00007691-198612000-00014.

[13] J. Ducharme, H. Fieger, M. Ducharme, S. Khalil, I. Wainer. Enantioselective disposition of hydroxychloroquine after a single oral dose of the racemate to healthy subjects. Br. J. Clin. Pharmacol. 40 (1995) 127-133. doi: https://doi.org/10.1111/i.1365-2125.1995.tb05768.x.

[14] S. Fu, A. Björkman, D. Ofori-Adjei, B. Wählin, O. Ericsson, F. Sjöqvist. In vitro activity of chloroquine, two enantiomers of chloroquine, desethylchloroquine and pyronaridine against Plasmodium falciparum, Br. J. Clin. Pharmac. 22 (1986) 93-96.

[15] G.B.A. Haberkorn, H.P. Kraft, G. Blaschke. Antimalarial activity in animals of the optical isomers of chloroquine diphosphate. Tropenmed Parasitol. 30 (1979) 308-312.

[16] R.F.J. Ducharme. Clinical Pharmacokinetics and Metabolism of Chloroquine. Clin. Pharmacokinet. 31 (2012) 2570274.

[17] I. D'Acquarica, I. Agranat. Chiral switches of chloroquine and hydroxychloroquine: potential drugs to treat COVID-19. Drug Discov. Today 25 (2020) 1121-1123. doi: https://doi.org/10.1016/j.drudis.2020.04.021.

[18] J. Iredale, I.W. Wainer. Determination of hydroxychloroquine and its major metabolites in plasma using sequential achiral-chiral high-performance liquid chromatography. J. Chromatogr. B Biomed. Sci. Appl. 573 (1992) 253-258. doi: https://doi.org/10.1016/0378-4347(92)80126-B.

[19] O. Walker, D. Birkett, G. Alvan, L. Gustafsson, F. Sjoqvist. Characterization of chloroquine plasma protein binding in man. Br. J. Clin. Pharmacol. 15 (1983) 375-377. doi: https://doi.org/10.1111/j.1365-2125.1983.tb01513.x.

[20] D. Ofori-Adjei, O. Ericsson, B. Lindstrom, F. Sjoqvist. Protein binding of chloroquine enantiomers and desethylchloroquine. Br. J. Clin. Pharmacol. 22 (1986) 356-358. doi: https://doi.org/10.1111/i.13652125.1986.tb02900.x.

[21] A. McLachlan, S. Tett, D. Cutler, R. Day. Disposition of the enantiomers of hydroxychloroquine in patients with rheumatoid arthritis following multiple doses of the racemate. Br. J. Clin. Pharmacol. 36 (1993) 78-81. doi: https://doi.org/10.1111/j.1365-2125.1993.tb05897.x.

[22] K.E. Ibrahim, A.F. Fell. Separation of chloroquine enantiomers by high-performance liquid chromatography. J. Pharm. Biomed. Anal. 8 (1990) 449-452. doi: https://doi.org/10.1016/07317085(90)80074-Y.

[23] A. Aubry, F. Gimenez, R. Farinotti, I.W. Wainer. Enantioselective Chromatography of the Antimalarial Agents Chloroquine, Mefloquine, and Enpiroline on a a1-Acid Glycoprotein Chiral Stationary Phase. Chirality 35 (1992) 3-8. doi: https://doi.org/10.1002/chir.530040108.

[24] G. Li, J. Sun, Y.Y. Huang, Y. Li, Y. Shi, Z. Li, X. Li, F.H. Yang, J. Zhao, H.B. Luo, T. Zhang, X. Zhang. Enantiomers of Chloroquine and Hydroxychloroquine Exhibit Different Activities Against SARS-CoV-2 in vitro, Evidencing S -Hydroxychloroquine as a Potentially Superior Drug for COVID-19. (2020). doi: https://doi.org/10.1101/2020.05.26.114033.

[25] Daicel Chiral Application, n.d. https://search.daicelchiral.com.

[26] K.L. Valko, S.P. Teague, C. Pidgeon. In vitro membrane binding and protein binding (IAM MB/PB technology) to estimate in vivo distribution: applications in early drug discovery. ADMET DMPK 5 (2017) 14. doi: https://doi.org/10.5599/admet.5.1.373.

[27] K.L. Valkó, S.B. Nunhuck, A.P. Hill. Estimating Unbound Volume of Distribution and Tissue Binding by in vitro HPLC-based Human Serum Albumin and Immobilized Artificial Membrane-Binding Measurements. J. Pharm. Sci. 100 (2011) 849-862. doi: https://doi.org/10.1002/jps.22323.

[28] K. Valko. Lipophilicity and biomimetic properties measured by HPLC to support drug discovery. $J$. Pharm. Biomed. Anal. 130 (2016) 35-54. doi: https://doi.org/10.1016/i.jpba.2016.04.009. 
[29] K. Valko, C. Du, C. Bevan, D. Reynolds, M. Abraham. Rapid Method for the Estimation of Octanol / Water Partition Coefficient (Log Poct) from Gradient RP-HPLC Retention and a Hydrogen Bond Acidity Term (Sigma alpha2H). Curr. Med. Chem. 8 (2001) 1137-1146. doi: https://doi.org/10.2174/0929867013372643.

[30] K. Valko, S. Nunhuck, C. Bevan, M. Abraham, D. Reynolds. Fast gradient HPLC method to determine compounds binding to human serum albumin. Relationship wth octanol water and immobilized artificial membrane lipophilicity. J. Pharm. Sci. 92 (2003) 2236-2248. doi: https://doi.org/10.1002/jps.10494.

[31] K. Valko. Physicochemical and biomimetic properties in drug discovery - Chromatographic techniques for lead optimization. Wiley, Hoboken, NJ, USA, 2014.

[32] C. Hansch, A. Leo. Exploring QSAR: Hydrophobic, Electronic, and Steric Constants. American Chemical Society, Washington, DC., 1995.

[33] D.C. Warhurst, J.C.P. Steele, I.S. Adagu, J.C. Craig, C. Cullander. Hydroxychloroquine is much less active than chloroquine against chloroquine-resistant Plasmodium falciparum, in agreement with its physicochemical properties. J. Antimicrob. Chemother. 52 (2003) 188-193. doi: https://doi.org/10.1093/jac/dkg319.

[34] Z. Jiang, J. Reilly. Chromatography approaches for early screening of the phospholipidosis-inducing potential of pharmaceuticals. J. Pharm. Biomed. Anal. 61 (2012) 184-190. doi: https://doi.org/10.1016/i.jpba.2011.11.033.

[35] Z. Yu, A.P. IJzerman, L.H. Heitman. Kv11.1 (hERG)-induced cardiotoxicity: A molecular insight from a binding kinetics study of prototypical Kv11.1 (hERG) inhibitors. Br. J. Pharmacol. 172 (2015) 940-955. doi: https://doi.org/10.1111/bph.12967.

[36] F. Barbato, V. Cirocco, L. Grumetto, M. Immacolata La Rotonda. Comparison between immobilized artificial membrane (IAM) HPLC data and lipophilicity in n-octanol for quinolone antibacterial agents. Eur. J. Pharm. Sci. 31 (2007) 288-297. doi: https://doi.org/10.1016/j.ejps.2007.04.003.

[37] R. Kaliszan, A. Nasal, M. Turowski. Binding-Site for Basic Drugs on Alpha(1)-Acid Glycoprotein as Revealed by Chemometric Analysis of Biochromatographic Data. Biomed. Chromatogr. 9 (1995) 211215. doi: https:/doi.org/10.1002/bmc.1130090504.

[38] Z.H. Israili, P.G. Dayton. Human alpha 1-acid glycoprotein and its interactions with drugs. Drug Metab. Rev. 33 (2001) 161-235. doi: https:/doi.org/10.1081/dmr-100104402.

[39] F. Hollosy, K. Valko, A. Hersey, S. Nunhuck, G. Keri, C. Bevan. Estimation of Volume of Distribution in Humans from HPLC Measurements of Human Serum Albumin Binding and Immobilized Artificial Membrane Partitioning. J. Med. Chem. 49 (2006) 6958-6971. doi: https://doi.org/10.1021/jm050957i.

[40] J.A. Shayman, A. Abe. Drug Induced Phospholipidosis: An Acquired Lysosomal Storage Disorder. Biochim. Biophys. Acta 1831 (2013) 602-611. doi: https://doi.org/10.1016/j.bbalip.2012.08.013.

[41] J. Müller-Höcker, H. Schmid, M. Weiss, U. Dendorfer, G.S. Braun. Chloroquine-induced phospholipidosis of the kidney mimicking fabry's disease: Case report and review of the literature. Hum. Pathol. 34 (2003) 285-289. doi: https://doi.org/10.1053/hupa.2003.36.

[42] J.-P.H.T.M. Ploemen, J. Kelder, T. Hafmans, H. van de Sandt, J.A. van Burgsteden, P.J.M. Salemink, E. van Esch. Use of physicochemical calculation of pKa and CLogP to predict phospholipidosis-inducing potential. Exp. Toxicol. Pathol. 55 (2004) 347-355. doi: https://doi.org/10.1078/0940-2993-00338.

[43] F. Hollósy, K. Valkó, A. Hersey, S. Nunhuck, G. Kéri, C. Bevan. Estimation of volume of distribution in humans from high throughput HPLC-based measurements of human serum albumin binding and immobilized artificial membrane partitioning. J. Med. Chem. 49 (2006) 6958-71. doi: https://doi.org/10.1021/im050957i.

[44] K. Valko, S. Rava, S. Bunally, S. Anderson. Revisiting the application of immobilized artificial membrane (IAM) chromatography to estimate in vivo distribution properties of drug discovery compounds based on the model of marketed drugs. ADMET DMPK 8 (2020) 78-97. doi: https://doi.org/10.5599/admet.757. 
[45] K. Valko, E. Chiarparin, S. Nunhuck, D. Montanari. In vitro measurement of drug efficiency index to aid early lead optimization. J. Pharm. Sci. 101 (2012). doi: https://doi.org/10.1002/ips.23305.

[46] L.J. Gordon, M. Allen, P.E.R. Artursson, M.M. Hann, B.J. Leavens, A. Mateus, S.I.M.O.N. Readshaw, K. Valko, G.J. Wayne, A.N.D.Y. West. Direct Measurement of Intracellular Compound Concentration by RapidFire Mass Spectrometry Offers Insights into Cell Permeability. J. Biomol. Screen. 21 (2016). doi: https://doi.org/10.1177/1087057115604141.

[47] S. Krishna, N.J. White. Pharmacokinetics of quinine, chloroquine and amodiaquine. Clinical implications. Clin. Pharmacokinet. 30 (1996) 263-299. doi: https://doi.org/10.2165/00003088199630040-00002.

[48] H.S. Lim, J.S. Im, J.Y. Cho, K.S. Bae, T.A. Klein, J.S. Yeom, T.S. Kim, J.S. Choi, I.J. Jang, J.W. Park. Pharmacokinetics of hydroxychloroquine and its clinical implications in chemoprophylaxis against malaria caused by plasmodium vivax. Antimicrob. Agents Chemother. 53 (2009) 1468-1475. doi: https://doi.org/10.1128/AAC.00339-08.

[49] L. Gustafsson, O. Walker, G. Alvan, B. Beermann, F. Estevez, L. Gleisner, B. Lindstrom, F. Sjoqvist. Disposition of chloroquine in man after single intravenous and oral doses. Br. J. Clin. Pharmacol. 15 (1983) 471-479. doi: https://doi.org/10.1111/j.1365-2125.1983.tb01532.x.

[50] S. Tett, D. Cutler, R. Day, K. Brown. A dose-ranging study of the pharmacokinetics of hydroxychloroquine following intravenous administration to healthy volunteers. Br. J. Clin. Pharmacol. 26 (1988) 303-313. doi: https://doi.org/10.1111/j.1365-2125.1988.tb05281.x.

[51] D.E. Furst. Pharmacokinetics of hydroxychloroquine and chloroquine during treatment of rheumatic diseases. Lupus 5 (1996) 11-15. doi: https://doi.org/10.1177/0961203396005001041.

[52] A.J. McLachlan, S.E. Tett, D.J. Cutler, R.O. Day. Disposition and absorption of hydroxychloroquine enantiomers following a single dose of the racemate. Chirality 6 (1994) 360-364. doi: https://doi.org/10.1002/chir.530060421.

[53] I.W. Wainer, J.C. Chen, H. Parenteau, S. Abdullah, J. Ducharme, H. Fieger, J. Iredale. Distribution of the enantiomers of hydroxychloroquine and its metabolites in ocular tissues of the rabbit after oral administration of racemic-hydroxychloroquine. Chirality 6 (1994) 347-354. doi: https://doi.org/10.1002/chir.530060419.

[54] P. Augustijns, N. Verbeke. Stereoselective Pharmacokinetic Properties of Chloroquine and De-EthylChloroquine in Humans. Clin. Pharmacokinet. 24 (1993) 259-269. doi: https://doi.org/10.2165/00003088-199324030-00007.

C2021 by the authors; licensee IAPC, Zagreb, Croatia. This article is an open-access article distributed under the terms and conditions of the Creative Commons Attribution license (http://creativecommons.org/licenses/by/4.0/) (cc)) EY 